\title{
Reliability Evaluation of Power Supply for More-Electric-Aircraft Based on Information Entropy
}

\author{
Xuanxuan $Q i^{1,{ }^{*}}$, Jian'an $C a o^{1}$ and Xiaojiao $L i^{1}$ \\ ${ }^{1}$ School of Electrical Engineering,Xi'an Jiaotong University,Xi' an 710049, China
}

\begin{abstract}
In order to overcome the shortcomings of traditional power supply reliability evaluation model of multi-electric aircraft in the process of multi-component system research, this paper introduces the approximate probability distribution of multi-component system by cross-entropy, it proposes a Monte Carlo method based on information entropy to evaluate the power supply reliability of multi-electric aircraft, and obtains the approximate probability distribution by differential evolution to make the reliability evaluation. The estimated variance is approximately zero. Finally, taking an aircraft power supply system as an example, the convergence and accuracy of several reliability analysis methods are compared and analyzed. The results show the superiority of this method.
\end{abstract}

\section{Introduction}

Multi-power aircraft technology integrates the power generation, power distribution and power consumption of aircraft into a unified system, and implements unified planning, unified management and centralized control of power generation, distribution and power consumption systems. Due to the increase in the number and variety of finished parts of multi-electric aircraft, the reliability model of the multi-electric aircraft becomes a key problem. Therefore, it is very important to establish an efficient reliability evaluation model for multi-electric aircraft power supply systems.

Authors in [1-2] introduce the reliability analysis model of multi-electric aircraft power system, and explains the importance of calculating the reliability of multi-electric aircraft power supply from various aspects. The traditional analysis method is to analyze the reliability of the aircraft power supply system by using the fault tree [3-6], and theoretically provide the aircraft reliability analysis results. However, with the increase in the number of components of the aircraft power supply system, the analysis of the fault tree method is more complicated, and it is difficult to evaluate the reliability of the entire power supply system. In order to improve the efficiency of the fault tree, the neighboring matrix algorithm [7-9] is used to look for the minimal segmental sets of the fault tree, which has the advantage of strong versatility and compensates for the deficiency of the fault tree to some extent. However, the increase in the number of components in the aircraft power supply system makes the analysis process of the algorithm complicated and the calculation time is long. Due to the improvement of computer computing power, the probability analysis method-Monte Carlo method [1012 ] is gradually applied to the reliability evaluation of complex systems, which is flexible and simple to implement. Due to the large number of system components and low failure rate, if the traditional Monte Carlo method is used to evaluate the reliability of the entire multi-electric aircraft power supply system, a large number of samples are needed, which will greatly reduce the efficiency of the algorithm.

In view of the above problems, the approximate probability distribution of the multi-element system is introduced by cross entropy [13-18]. The approximate probability distribution can make the reliability evaluation variance zero, which improves the sampling efficiency. Finally, a case-by-case example is used to verify the superiority of the Monte Carlo method based on information entropy in the reliability evaluation of multi-electric aircraft power supply system.

\section{Power supply system and reliability model}

The power supply modes of multi-electric aircraft mainly include main power supply system and reserve power supply system. The main power supply system also contains two subsystems that are mutually hot standby. In Fig.1, $S_{1}$ and $S_{2}$ are parallel main power supply systems that are alternate with each other. No matter which subsystem is faulty, the other system will switch to the load supply by itself. $S_{3}$ is a reserve power supply system. When both $S_{1}$ and $S_{2}$ fail, switch to $S_{3}$ to supply power to the load. $Z_{1}, Z_{2}, Z_{3}, Z_{4}$, and $Z_{5}$ represent the secondary exciter,exciter,generator,inverter and controller, respectively. The protection devices in each power supply mode are $C_{1}, C_{2}$, and $C_{3}$. To ensure the reliability of the switching system power supply mode, the switching devices between the power generation systems are $B_{1}$ and $B_{2}$.

\footnotetext{
* Corresponding author: hfut_qxx@163.com
} 


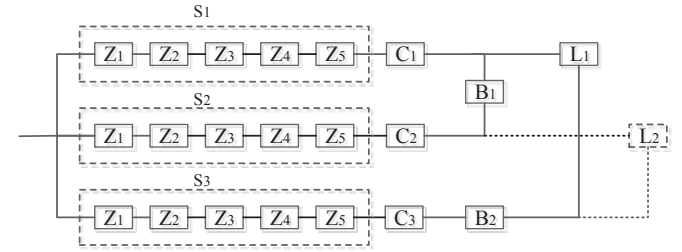

Fig.1 Aircraft power supply system structure

The reference value of the component probability density distribution is given in [9]. Since $Z_{1}, Z_{2}$, and $Z_{3}$ are related to the motor, it is assumed that their failure probability density function obeys a normal distribution. $Z_{4}, Z_{5}$ are fundamentally electronic devices, so they are assumed to follow an exponential distribution. Circuit breakers $C_{1}, C_{2}$ and $C_{3}$, contactors $B_{1}$ and $B_{2}$ are assumed to also follow an exponential distribution. To simplify the description of this model, each load in the system can be grouped into a load. This paper assumes that the set of all loads in the system is $L_{I}$. The aircraft power supply system has many components and its state model is different. For the convenience of modeling, this paper has done a simplification process, assuming that the state model of each component in the system is a two-state model.

The fault probability density function of the component is $f_{i}(t), i=1,2 \ldots 10$. The probability density function of the component is integrated to derive the probability of failure of the component, and the reliability of the component is determined.

$$
R_{i}(t)=1-F_{i}(t)=1-\int_{0}^{t} f_{i}(t) d t
$$

According to the reliability calculation criterion, the reliability of the series system is the reliability accumulation of each series element, so the reliability function expression of each subsystem is obtained as follows.

$$
R_{s_{n}}(t)=\prod_{i=1}^{5} R_{i}(t) \quad n=1,2,3
$$

Since the three systems are connected in parallel, the failure probability of the parallel system is the cumulative probability of failure of the three subsystems. First, obtain the probability of system failure, and then use one to get the reliability model of the whole system.

$$
R(t)=1-\prod_{i=1}^{3}\left(1-R s_{i}(t)\right)
$$

Through the above derivation, the analytical expression of the system reliability of the simplified aircraft power supply system model can be obtained. Since the probability distribution of each component is different, the formula (2) is accumulated after integration, so the complexity is high. To further introduce system reliability, the complexity of equations (3) is even higher. Formula (3) is applicable to the simplified model of aircraft power supply system in this paper. When the number of system components increases and the system structure is not simply seriesparallel, it will be difficult to find an analytical expression such as equation (3). Therefore, this paper will avoid the analytical method to calculate system reliability. Monte Carlo is used to sample aircraft system fault conditions for the current short time range. At the same time, the method of information entropy is introduced to optimize the sampling probability density function of the sample. The probability density function can effectively solve the problem of low efficiency of traditional Monte Carlo method.

\section{Establishment of multi-electric aircraft estimation model}

Because of the high reliability of the aircraft power supply system, it is difficult to sample the system fault components during Monte Carlo sampling, so the calculated system reliability is generally 1 . This is obviously not what this article needs. In order to improve the sampling efficiency, the distribution of the probability $f(x, u)$ of the original system is improved to the optimal probability distribution $g(x)$, so that the coefficient of variance can be theoretically zero. But $g(x)$ is often difficult to find, or even impossible to solve. For the reason that, this paper introduces the cross entropy method, which is generally used to measure the gap between the target distribution and the predicted value distribution. Therefore, this paper constructs a probability distribution close to the $g(x)$ distribution by the method of cross entropy.

The reliability evaluation expression of the aircraft power supply system is as follows:

$$
l=\int I_{s(x)<r} f(x, u) d x \approx \frac{1}{N} \sum_{i=1}^{N} I_{\mathrm{S}(X)<r}
$$

In formula (4), $f(x, u)$ represents the probability density function of the state of the system component. In order to improve the sampling efficiency, it is necessary to deform the formula (4). The probability density after deformation can make the reliability variance coefficient zero.

$$
l=\int I_{s(x)<r} \frac{f(x, u)}{g(x)} g(x) d x=\int L(x) g(x) d x
$$

In formula (5), $L(x)$ is the ratio between the original probability density function of system components and the improved probability density function, which is often called likelihood ratio. $g(x)$ is an arbitrary probability density function. The Monte Carlo estimate for equation (5) is:

$$
\hat{l}=\frac{1}{N} \sum_{i=1}^{N} I_{S\left(X_{i}\right)<r} \frac{f\left(X_{i}, u\right)}{g\left(X_{i}\right)}
$$

In equation (6), $X_{i}$ is the state of the system component obtained by sampling one time under the probability density function $g(x)$.

When $g(x)$ is equal to $g_{\text {opt }}(x)$, the system reliability estimation variance of $l$ is zero. The expression of $g_{\text {opt }}(x)$ is as in formula (7).

$$
g_{\text {opt }}(x)=I_{S(x)<r} \frac{f(x, u)}{l}
$$

It can be seen that the original probability density function divided by $l$ can make the variance of the reliability estimate be zero. Therefore, when Monte Carlo sampling, $N$ samples can be randomly sampled 
under the $g_{\text {opt }}(x)$ probability density function. However, it can be seen from equation (10) that $g_{\text {opt }}(x)$ is related to $l$, but $l$ is the reliability to be determined. So it is unrealistic to use the $g_{\text {opt }}(x)$ probability density function directly.

This paper introduces the probability density function $f(x, v)$ as an approximate probability density function of $g_{\text {opt }}(x)$. In order to measure the closeness of $f(x, v)$ and $g_{\text {opt }}(x)$, this paper uses cross entropy to measure the distance between the target distribution and the approximate probability distribution. As shown in formula (8).

$$
D=\int g(x) \ln (g(x)) d x-\int g(x) \ln (f(x, v)) d x
$$

Minimizing the closeness of $f(x, v)$ and $g_{\text {opt }}(x)$ is to find the minimum value of $D$, that is, to find the minimum value of $-\int g_{\text {opt }}(x) \ln (f(x, v)) d x$. Finally, it is equivalent to the maximum value of the formula (9).

$$
\max _{v} \int g_{\text {opt }}(x) \ln (f(x, v)) d x
$$

Substitute $g_{\text {opt }}(x)$ in formula (7) into formula (9) to get formula (10).

$$
\max _{v} \int \frac{I_{\{S(x) \leq r\}} f(x, u)}{l} \ln (f(x, v)) d x
$$

Since $l$ is constant for known systems. Therefore, the equivalent estimates of equation (10) are as follows:

$$
\max _{v} E_{U}\left(I_{\{S(X) \leq r\}} \ln (f(X, v))\right)
$$

The above derivation is based on the probability density function. Since the variables in this paper are discrete, it is necessary to convert the probability density function into a probability distribution function. For example, $f(x, u)$ is converted to $F(x, u), f(x, v)$ is converted to $F(x, v)$, and the integral becomes a summation.

$X$ is a sample of the system sampled from the probability distribution function $F(X, u)$. Since the sampling of $F(x, u)$ yields only a small number of valid samples, the important sampling method is used to replace $F(x, u)$ with $F(x, w)$. Therefore, the formula (11) is transformed into the formula (12).

$$
\max _{v} E_{w} I_{\{S(X) \leq r\}} W(X ; u, w) \ln (F(X, v))
$$

$X$ is a sample of the system sampled from the probability distribution function $F(X, w)$. Where $W(X ; u, w)$ $=F(x, u) / F(x, w)$. The optimal probability distribution function $F(x, v)$ of the system can be obtained by solving the maximum value of the formula (12). It can be seen that the maximum solution of formula (12) is a multivariable maximum optimization process. In this paper, differential evolution [19-21] is introduced to iteratively solve $F(x, v)$. Then the samples are sampled under the new probability distribution function, and the system reliability index is solved according to the formula (6).

In order to further improve the sampling efficiency, one-time sampling using dual sampling [22-24] produces random numbers that are negatively correlated with each other. This feature of the negative correlation between related points is used to quickly reduce the variance of the estimated value.

\section{Simulation and Verification}

\subsection{Instantiated System Estimation Model}

Table 1 shows the distribution types and parameters of the various components of the power supply system under the two system models. The remaining components not given are considered to be very reliable.

In this paper, the test function $I S(X)<r$ represents the system fault condition. When $S(X)<r$ is zero, it represents fault, and $S(X)>r$ is one for operation. $S(X)$ represents the generating capacity of aircraft power supply system, and $r$ represents aircraft load. The number of system components is $n$. It is specified that $X_{k}$ represents the $k$ th value in the system state space vector $\mathbf{X}, X_{k}=\left[x_{1}, x_{2}, x_{3} \ldots x_{n}\right]$. The system component is a twostate model and $u_{i}$ is the probability of failure of component $i$. The specific value of $u_{i}$ is sampled according to the failure probability distribution of the component. The probability distribution function $F(x, u)$ of the system is derived from the above assumptions.

$$
F(x, u)=\prod_{i=1}^{n}\left(1-u_{i}\right)^{1-x_{i}}\left(u_{i}\right)^{x_{i}}
$$

Table 1 Failure distribution parameters

\begin{tabular}{|c|c|c|c|}
\hline unit & $\begin{array}{c}\text { distribution } \\
\text { pattern }\end{array}$ & $\begin{array}{c}\text { A system } \\
\text { parameters }\end{array}$ & $\begin{array}{c}\text { B system } \\
\text { parameters }\end{array}$ \\
\hline $\mathrm{Z} 1$ & Normal & $u_{1}=1500 ; \sigma_{1}=120$ & $u_{1}=3750 ; \sigma_{1}=600$ \\
\hline $\mathrm{Z} 2$ & Normal & $u_{2}=1500 ; \sigma_{2}=150$ & $u_{2}=3750 ; \sigma_{2}=750$ \\
\hline $\mathrm{Z} 3$ & Normal & $u_{3}=2800 ; \sigma_{3}=500$ & $u_{3}=7000 ; \sigma_{3}=1250$ \\
\hline $\mathrm{Z} 4$ & exponential & $\lambda_{4}=1.25 \times 10^{-3}$ & $\lambda_{4}=5 \times 10^{-4}$ \\
\hline $\mathrm{Z} 5$ & exponential & $\lambda_{4}=1.25 \times 10^{-3}$ & $\lambda_{4}=5 \times 10^{-4}$ \\
\hline
\end{tabular}

Since $S(X)<r$ is a small probability event, the probability distribution function $F(x, u)$ is changed to $F(x, v)$ by cross entropy. The system reliability estimate under the improved probability distribution function $F(x, v)$ is shown in equation (14).

$$
l=\frac{1}{N} \sum_{i=1}^{N} I_{S(X)<r} W(X, u, v)
$$

Where $W(X, u, v)$ is the correction factor. The specific form is as follows:

$$
W(X, u, v)=\frac{\prod_{i=1}^{n}\left(1-u_{i}\right)^{1-x_{i}}\left(u_{i}\right)^{x_{i}}}{\prod_{i=1}^{n}\left(1-v_{i}\right)^{1-x_{i}}\left(v_{i}\right)^{x_{i}}}
$$

The change in state space probability is determined by the parameter vector $\mathbf{v}=\left[v_{1}, v_{2}, v_{3} \ldots v_{n}\right]$. The problem now is to look for the optimal $\mathbf{v}$ to find $F(x, v)$. In this paper, the differential evolution algorithm is used to solve the optimal value $\mathbf{v}$. Differential evolution is characterized by simple structure, superior performance, and parallelism of algorithms, which can be searched collaboratively. The objective function can be optimized quickly and concisely. 


\subsection{Implementation process}

\subsubsection{Parameter initialization}

Initialize parameter vector $\mathbf{v}$, optimize process sample size $N$, system component failure rate $u_{i}$, failure rate scaling factor $k$ and differential evolution iteration number $N_{s}$. The sampling sample size of the dual variable method $N_{d}$, the variance convergence coefficient $\beta$.

\subsubsection{Determine the optimization objective function}

$\mathrm{N}$ samples are randomly sampled according to the system component failure rate $u_{i}$ and the failure rate scaling factor $k$. The optimization objective function is determined according to the formula (12).

\subsubsection{Variations}

The $i_{\text {th }}$ individual variation in a population. Individual variation is realized by difference strategy.

\subsubsection{Cross}

The purpose of the crossover operation is to randomly select individuals and determine whether to accept the variation results of the previous step.

\subsubsection{Screening}

The greedy selection strategy is adopted, which is to select the better individual as the new individual according to the value of the objective function.

\subsubsection{Conditional judgment}

Determine whether the number of differential evolution iterations reaches $N_{s}$. If it reaches the next step, the system probability distribution function is $F(x, v)$, otherwise it jumps back to step 4.2.2.

\subsubsection{Dual variable sampling}

Generate $n$ sample arrays in the interval [0,1], and subtract the array from 1 to get its dual array. The state of the components of the sampling system is obtained by the formula (4).

\subsubsection{Calculating the reliability index}

Calculate the reliability indicator according to formula (12).

\subsubsection{Conditional judgment}

Determine whether the variance coefficient satisfies the convergence condition. If it is satisfied, jump out of the loop and output related information and graphics, otherwise skip to step 4.2.7.

\subsection{Analysis of simulation results}

In order to verify the superiority of the algorithm in this paper, the traditional monte carlo method, analytical method and reliability evaluation method based on information entropy were used to simulate the calculation of A and B systems.

In order to verify the convergence characteristics of the proposed algorithm, this paper compares the convergence characteristics of the variance coefficients of the Monte Carlo method and the information entropybased reliability evaluation method. This stage simulates the system reliability at $1000 \mathrm{~h}$. At the same time, in order to ensure the accuracy of the calculation results, this paper carried out three reliability evaluations for the A system. Then take the average of 3 results as the final result. The Fig.below is the result of simulation with MATLAB.

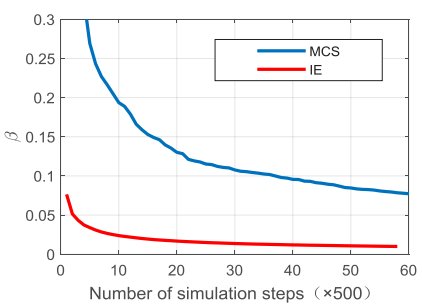

Fig.2 Convergence coefficient convergence graph

It can be seen from Fig.2 that the reliability evaluation method based on information entropy has very good convergence characteristics when evaluating the reliability of the aircraft power supply system. This method can achieve good convergence stability when the number of simulation times is 10000, while the traditional sampling method can achieve good convergence stability when the number of simulation times is 30000 . In comparison, the algorithm proposed in this paper improves the convergence by 2 times.

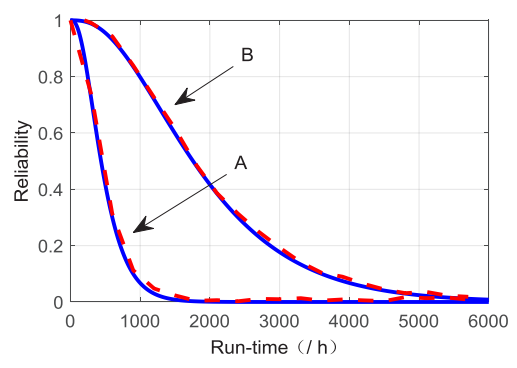

Fig.3 reliability of power system

In order to verify the accuracy of the proposed algorithm, this paper analyzes the reliability and timevarying characteristics of the analytical method and the reliability evaluation method based on information entropy.

Fig.3 shows the distribution of power system reliability over time. The red line represents the method of this article and the blue line represents the traditional analytical method. Fig. 4 is the error distribution diagram between the reliability evaluation method based on information entropy and the traditional analytical method in a simulation. For the A power system, the maximum error of the method proposed in this paper is 0.02 
compared with the traditional method, and the maximum proportion is $2 \%$. For the $\mathrm{B}$ power system, the maximum error of the method in this paper compared with the traditional method is 0.018 , and the maximum proportion is $1.8 \%$.

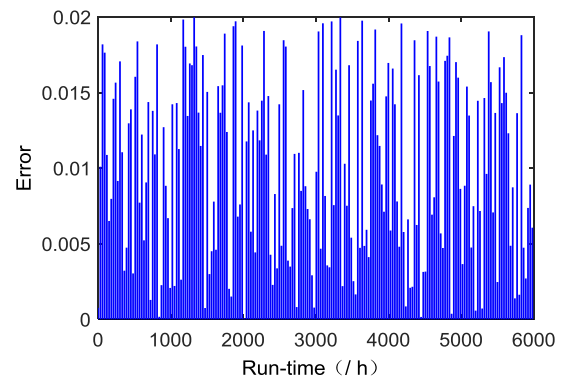

Fig.4 error distribution

From the above analysis, it can be concluded that for the reliability analysis of the power system, the analysis results of the reliability estimation model obtained by the information entropy-based reliability evaluation method are more convergent than the reliability analysis results directly obtained by Monte Carlo simulation. Well, compared with the reliability analysis results obtained by the traditional analytical method, the accuracy of the calculation results is relatively close, and the computational model is less complex. Therefore, the model can meet the requirements of aviation power system reliability estimation.

\section{Conclusion}

This paper uses information entropy-based reliability assessment to assess the reliability of complex systems. And the probability density distribution is introduced by using cross entropy, so that the reliability variance is theoretically zero. Then it uses the differential evolution approximation probability function. Finally, under the approximate probability distribution, combined with the dual sampling method, the reliability of the whole system is evaluated. At the end of the paper, an example is used to evaluate the reliability of the method using the analytical method, the Monte Carlo method and the information entropy-based reliability evaluation method. From the convergence analysis, the convergence of this method is more than 2 times compared with the reliability analysis results obtained by direct Monte Carlo simulation. From the calculation accuracy analysis, compared with the reliability analysis results obtained by the traditional analytical method, the accuracy of the calculation results is relatively close, but the computational model is less complex. Therefore, the comprehensive analysis of this method is very suitable for the more complex reliability evaluation of aircraft power system.

\section{References}

1. Yiping Zhang. Research on Aircraft Low Voltage DC Power System.(2001)

2. Yi Ming. Aircraft power system[M],(2013).
3. Tao Cao, Shanyong Wu, Junhua Fang. Reliability Analysis of Aircraft Power System Based on Fault Tree .The 9th Yangtze River Delta Science and Technology Forum - Proceedings of Aerospace Science and Technology Innovation and Yangtze River Delta Economic Transformation Development Sub-forum.(2012)

4. Haitao Bo, Zhenxing Bai. Fault diagnosis of aircraft power system based on fault tree and neural network. Modern Electronic Technology, vol.28, no.10, p.65-67, (2005)

5. Xiaojing Guo, Yunlin Lin. Research on Aircraft TCAS Fault Diagnosis Based on Fault Tree and Neural Network[C]. China Academic Conference on Control and Decision Making.(2006)

6. Hengcheng $\mathrm{Xu}, \mathrm{Pu}$ Chen. Logical Analysis of Aircraft Power System Fault Diagnosis Using Computer Technology. Foreign electronic measurement technology.vol.22, no.3, p.15-17, (2003)

7. Zongxiang Lu, Yongji Guo. Reliability evaluation of electrical main wiring of hydropower station[J]. Power System Automation. vol. 18, p. 16-19,(2001)

8. Hongjiang $\mathrm{Li}$, Zongxiang Lu, Miao Wang. Comparative analysis of ship network topology based on reliability model[J]. Transactions of China Electrotechnical Society, vol 21, no 11, p. 16-19, (2001)

9. Chiquet, Julien, Mohamed Eid, and Nikolaos Limnios. "Modelling and estimating the reliability of stochastic dynamical systems with Markovian switching." Reliability Engineering \& System Safety . vol 93, no.12, p. 1801-1808, (2008)

10. Jirutitijaroen, Panida, and Chanan Singh. "Comparison of simulation methods for power system reliability indexes and their distributions." IEEE Transactions on Power Systems vol.23, no.2 , p. 486-493, (2008).

11. Hongzhe Dai, WeiWang.Quasi-Monte Carlo method for structural reliability analysis. Journal of Aviation.vol.30, no. 4, p.666-671,(2009)

12. WeiWang, MingShun Song, Yihua Chen. Monte Carlo method for the evaluation of uncertainty in measurement of complex models. Journal of Instrumentation.vol. 29, no 7, (2008)

13. Ni Chen, Qian Sha, Yi Tang. Wind power combination forecasting method based on cross entropy theory. Proceedings of the CSEE. vol. 32, no. 2, p.29-34.(2012)

14. Ran Li, Huilan Liu,Yunlu. Combined prediction method of distribution transformer life based on cross entropy theory. Power system protection and control. no. 4, p. 97-103,(2014)

15. Yonghong Li,Yunqin Wu,Liang Ao. Reliability Evaluation Technology Based on Cross Entropy. Aviation Standardization \& Quality.no. 2, p. 37-30, (2010) 
16. Pengcheng Xu,Qi Chen,Wenxia Liu. Reliability assessment of wind power grids with cross entropy and dynamic fault set. Automation of Electric Power Systems. no. 13, p. 28-34,(2016)

17. Shaowei Wang,Pingping Luo. Reliability Evaluation of Power Generation System Based on Hybrid Model and Cross Entropy Importance Sampling. Electric Measurement \& Instrumentation.vol.54, no.3, p.35-41,(2017)

18. Xiang Zhao, Hongshuang Li. Global Reliability Sensitivity Analysis Based on Cross Entropy and Space Segmentation. Journal of Aviation.nol.39, no.2, p.179-189,(2018)

19. Lianghong Wu, Yaonan Wang, Xiaofang Yuan. Adaptive quadratic mutation differential evolution algorithm. Control and decision. Vol.21, no.8, p.898-902,(2006)

20. Shuben Zhao, Fusheng Zhang, Jiyou Zhong. Application of Adaptive Differential Evolution
Algorithm in Reactive Power Optimization of Power System. Power grid technology.vol.34, no.6, p.169-174,(2010)

21. Ying Huang, Yang Li, Ciwei Gao. Multi-objective power grid planning based on non-dominated sorting differential evolution algorithm. Power System Technology. no,3. p.85-89,(2011)

22. $\mathrm{Na} \mathrm{Yu,} \mathrm{Xiaotong} \mathrm{Li,} \mathrm{Tao} \mathrm{Meng.} \mathrm{Reliability}$ Assessment of Active Power Distribution System Based on Dual Sampling Monte Carlo Method. Power capacitor and reactive power compensation. vol,38. no,4. P177-182,(2017)

23. Yanhua Wei, Bingcan Wang,Yongzhong Xing. Monte Carlo integration method and its improvement. Statistics and Decision.no.12, p.1015,(2017)

24. Jianhau Wang. Reliability Analysis of Initial Support of Highway Tunnel Based on Monte Carlo Method. Road tunnel.no.12, p.14-17,(2007) 